\title{
Genome-wide analysis of the phospholipase D family in Oryza sativa and functional characterization of PLD $\beta 1$ in seed germination
}

\author{
Gang $\mathrm{Li}^{1}$, Fang Lin ${ }^{1,2}$, Hong-Wei Xue ${ }^{1}$ \\ ${ }^{I}$ National Key Laboratory of Plant Molecular Genetics, Institute of Plant Physiology and Ecology, Shanghai Institutes for Biological \\ Sciences, Chinese Academy of Sciences, 300 Fenglin Road, Shanghai 200032, China
}

Phospholipase D (PLD) plays a critical role in plant growth and development, as well as in hormone and stress responses. PLD encoding genes constitute a large gene family that are present in higher plants. There are 12 members of the PLD family in Arabidopsis thaliana and several of them have been functionally characterized; however, the members of the PLD family in Oryza sativa remain to be fully described. Through genome-wide analysis, 17 PLD members found in different chromosomes have been identified in rice. Protein domain structural analysis reveals a novel subfamily, besides the C2-PLDs and PXPH-PLDs, that is present in rice - the SP-PLD. SP-PLD harbors a signal peptide instead of the $\mathrm{C} 2$ or PXPH domains at the N-terminus. Expression pattern analysis indicates that most PLD-encoding genes are differentially expressed in various tissues, or are induced by hormones or stress conditions, suggesting the involvement of PLD in multiple developmental processes. Transgenic studies have shown that the suppressed expression of rice PLD 1 results in reduced sensitivity to exogenous ABA during seed germination. Further analysis of the expression of ABA signaling-related genes has revealed that PLD $\beta 1$ stimulates ABA signaling by activating $S A P K$, thus repressing GAmyb expression and inhibiting seed germination.

Keywords: Oryza sativa, PLD $\beta 1, \mathrm{ABA}$, seed germination

Cell Research (2007) 17:881-894. doi: 10.1038/cr.2007.77; published online 18 September 2007

\section{Introduction}

Phospholipase D (PLD), which hydrolyzes phospholipids to produce phosphatidic acid (PA) and a free head group such as choline, has been detected in bacteria, fungi, plants and animals [1]. Although PLD was found to be involved in lipid metabolism and membrane reconstruction in the 1940s [2], the first eukaryotic cDNA of PLD was cloned from the castor bean only in 1994 [3]. Since then, many PLD-encoding genes have been cloned from Arabidopsis

\footnotetext{
Correspondence: Hong-Wei Xue

Tel: +86 21 54924059; Fax: +862154924060

E-mail: hwxue@sibs.ac.cn

${ }^{2}$ Current address: Department of Pharmacology, Laboratory of Aging and Nervous Diseases, Soochow University School of Medicine, Suzhou 215123, China

Received 1 February 2007; revised 10 May 2007; accepted 4 June 2007; published online 18 September 2007
}

thaliana [4], Oryza sativa [5, 6], Zea mays [5], Nicotiana tabacum [7] and Lycopersicon esculentum [8]. Gene expression studies, protein domain structure analyses and biochemical characterization have greatly expanded our knowledge of the physiological functions and relevant regulations of PLD [9].

Biochemical studies have indicated that the phospholipid-hydrolyzing activities of PLD are either calcium-dependent (C2-PLD) or calcium-independent (PXPH-PLD) [10]. Various phospholipid molecules, including phosphatidylinositol (PI), phosphatidylcholine (PC), phosphatidylethanolamine (PE), phosphatidylglycerol (PG) and phosphatidylserine (PS), may be selectively hydrolyzed by different PLD members [11]. Protein domain analysis has resulted in the identification of several conserved domains, including (1) the PLD-C1 and PLD-C2 domains, which are also known as the HKD $(\mathrm{HxKxxxxD)}$ domains and are responsible for the hydrolysis activity; (2) the $\mathrm{C} 2$ domain, 
the calcium/lipid-binding domain that is responsible for regulating $\mathrm{Ca}^{2+}$-dependent activity through binding to $\mathrm{Ca}^{2+}$; and (3) the PX (phox consensus sequence) and $\mathrm{PH}$ (pleckstrin homology) domains, which are located at the $\mathrm{N}$-terminus of $\mathrm{Ca}^{2+}$-independent PLDs in place of the $\mathrm{C} 2$ domain of $\mathrm{Ca}^{2+}$-dependent PLDs [10].

Physiological studies have shown that PLDs are involved in multiple plant growth and developmental processes, such as seed germination, seedling growth, pollen tube germination and elongation, and leaf senescence $[12,13]$. Treatment with the PLD-specific inhibitor 1-butanol results in inhibited seed germination, altered emergence of the radicle and cotyledons, and abnormal root elongation and pattern formation of root hairs $[14,15]$. It was also shown that PLD and PA are critical for pollen germination and pollen tube elongation; reduced production of PA by PLD resulted in inhibited pollen tube germination and tip growth, and altered apical polarity of the pollen tube [16]. In addition, the actin and microtubule structures were severely altered under 1-butanol treatment [14, 17-19].

Additionally, PLD and its product PA mediate the signaling of various plant hormones, including abscisic acid [20-23], gibberellin [21], ethylene [20], cytokinin [24], jasmonic acid (JA) [25] and auxin [26]. In Arabidopsis, treatment with 1-butanol represses cytokinin-induced ARR5-GUS expression [24] and suppresses the auxin response [26]. Arabidopsis PLD $\alpha 1$ activity and PLD $\beta 1$ expression are wound-induced, and PLD $\alpha 1$ has been proven to be involved in ABA signal transduction and wound-induced JA accumulation [20, 25].

ABA is crucial in seed maturation, dormancy, germination and the cell response to stress [27]. Recent genetic and biochemical approaches have enabled the identification of numerous components that are involved in ABA signaling, such as the ABA receptors FCA [28] and ABAR/CHLH [29], ABI1 [30, 31], G protein [32] and PLD [21, 22, 33, 34]. PLD and PA play an important role in ABA signaling. Suppressed PA or extra PA supply is able to either counteract or mimic the effects of ABA in barley aleuronic cells [21] and Vicia faba guard cells [33]. Indeed, it has been shown that ABA stimulates PLD activity and increases the amount of PA produced [21, 23, 35, 36]. In barley aleurone cells, PLD and PA mediate the inhibitory effects of ABA on GA-promoted $\alpha$-amylase production [21]. In Arabidopsis, the amount of PA is increased at an early stage of seed germination, and increased levels of PA through a deficiency in lipid phosphate phosphatase (LPP) lead to the reduced conversion of PA to diacylglycerol (DAG), which results in hypersensitive responses to ABA during seed germination [23]. This is consistent with a previous report that the repressed expression of $P L D \alpha 1$ results in a decreased sensitivity to ABA and drought-induced stomatal closure [37].

Recent studies have shown that PLD and PA mediate plant responses to $\mathrm{ABA}$ by regulating the localization and activity of ABI1 [22], a negative regulator of the ABA signaling pathway $[30,31]$. PA tethers $A B I 1$ to the plasma membrane, which results in reduced translocation of ABI1 to the nucleus, and decreases ABI1 phosphatase activity [22]. In addition, PLD $\alpha 1$ and PA are involved in ABA signaling through interaction with GPA1, a heterotrimeric GTP-binding protein (G protein) [34, 38].

Genome-wide analysis identified 12 PLD members in Arabidopsis [10, 39], and several members, including PLD $\alpha 1, \operatorname{PLD} \delta, \operatorname{PLD} \zeta 1$ and PLD $\zeta 2$, were studied using biochemical or physiological approaches. PLD $\alpha 1$, the predominant PLD, is responsible for the common PLD activity in Arabidopsis, and is involved in ABA and ethylene signal transduction, freezing tolerance and wound-induced JA accumulation [13]. PLD $\beta 1$ can bind to $\alpha$-actin in vitro [40], and its expression is induced by wound stress [25]. PLD $\delta$ is activated by oleic acid and is closely associated with the microtubule cytoskeleton and plasma membrane. It plays a positive role in the plant's response to different environmental stresses such as freezing and oxidative assault [13]. $P L D \zeta 1$ is involved in root hair pattern formation, and is a direct target of the homeobox transcription factor GLABRA2 (GL2) [15]. Root elongation and digalactosyldiacylglycerol accumulation during phosphorus-limited growth conditions can be affected by PLD $\zeta 2$ [41, 42]. Recently, our studies have shown that $P L D \zeta 2$ expression is induced by IAA, and is required for the auxin response [26].

Environmental stimuli, such as drought and phosphate starvation, are critical factors affecting crop production, and the involvement of PLDs in these processes suggests their functional importance in crop growth. However, the presence and function of PLDs in rice, the model species for crops, remain to be fully studied. Here we present a detailed analysis of PLD genes in rice, and their phylogenetic relationship with their orthologs in Arabidopsis. In addition, our physiological studies showed that $P L D \beta 1$, a C2-PLD, is involved in seed germination through mediating ABA signal transduction.

\section{Materials and Methods}

\section{Enzymes and chemicals}

Enzymes used for DNA restriction and modification were obtained from Boehringer (Mannheim, Germany). IAA, GA, ABA and PA were obtained from Sigma-Aldrich (St Louis, MO, USA). The 'Trizol Kit' for RNA extraction was obtained from Invitrogen Company. DNA primers for polymerase chain reaction (PCR), Taq polymerase and a 'random labeling' kit were obtained from Genecore (Shanghai, China) and TaKaRa Biotechnology (Dalian, China). Nylon membranes and radiochemical $\left[\alpha-{ }^{32} \mathrm{P}\right] \mathrm{dCTP}$ were obtained 
from Amersham Pharmacia Biotech (USA) and Yahui Company (Beijing, China).

\section{Bacteria and plant material}

Escherichia coli DH5 $\alpha$ cells were used for amplifying the cDNA library. The Agrobacterium tumefaciens EHA105 strain was used for rice transformation. Oryza sativa $c v$. Zhonghua 11 were germinated on $1 / 2 \mathrm{MS}$ medium and grown in water in a phytotron with a 12-h light $\left(26^{\circ} \mathrm{C}\right)$ and 12 -h dark $\left(18^{\circ} \mathrm{C}\right)$ period. For hormone and stress treatments, 2-week-old rice seedlings were treated with various hormones or stress conditions for 3 or $6 \mathrm{~h}$ (IAA, $100 \mu \mathrm{M}$; GA, $100 \mu \mathrm{M}$; ABA, $\left.100 \mu \mathrm{M} ; \mathrm{NaCl}, 250 \mathrm{mM}, 29^{\circ} \mathrm{C}\right)$.

\section{Database search and sequence analysis}

To identify members of the rice PLD family, multiple database searches were performed. First, we carried out a BLAST search of the TIGR rice annotation database (http://www.tigr.org/rice, Release 4), querying with the Arabidopsis PLD sequences. We also searched the annotation database using the gene name and conserved HKD domain (PF00614, PLD active site motif) as keywords. BLAST and keyword searches with the gene name were done for the Rice Annotation Project database (RAP, http://rapdb.lab.nig.ac.jp/cgibin/gbrowse/IRGSP, Build 4.0). In addition, the GRAMENE Rice Protein Database (http://www.gramene.org/) and the National Center for Biotechnology Information's GenBank (http://www.ncbi.nlm. nih.gov) were searched to identify $P L D$ genes in rice. All the $P L D$ genes identified have corresponding gene names in three other public rice databases, so the corresponding DNA and predicted PLD protein sequences from various database annotations were checked using ClustalW, with the default parameters as set by the European Bioinformatics Institute (EBI, http://www.ebi.ac.uk/clustalw/index. $\mathrm{html}$ ). Finally, based on the protein sequence comparisons, the conserved domain composition and the phylogenetic relationship with Arabidopsis PLDs, all the rice PLD family genes were classified into different subgroups, and renamed with the uniform name.

The total number of ESTs was calculated from a GenBank database BLAST search. Hits from BLAST searches that had E-values above 0.1 were not considered for further analysis. The exon-intron structures of $P L D$ genes were taken from the TIGR database and confirmed by comparing the cDNA with the corresponding genomic sequences. Domain and motif searches were carried out on the protein sequences in SMART (http://smart.embl-heidelberg.de/) and Pfam (http://www.sanger.ac.uk/software/pfam). Searching for targeting signals was performed using the TargetP program (http://www.cbs. dtu.dk/services/TargetP).

\section{Expression pattern analysis via quantitative reverse transcrip- tion (RT)-PCR}

Total RNA was isolated from seedlings, roots, stems, leaves and immature seeds or from harvested material treated with plant hormones and environmental stimuli. In all, $2 \mu \mathrm{g}$ of total RNA was reverse transcribed according to the supplier's instructions (ReverTra plus, TOYOBO, Japan) and real-time RT-PCR was executed using the Rotor-Gene 3000 (Corbett Research, Sydney, Australia) with an SYBR green probe (SYBR Premix Ex Taq system, Takara). The amounts of amplified product were determined at the end of each cycle using the Rotor-Gene software (Ver. 6.0.16, Corbett Research). Rice actin1 (OSRAC1, X16280) was used as the internal positive control. The DNA primers used for qRT-PCR are listed as follows:
PLD $\alpha 1-S$ (5'-TGG GTA ACC GTG AGG TGA AGC AG-3') and PLD $\alpha 1$-A (5'-CCA TGG CGA TCT CAG AGT CCC TAG-3'); PLD $\alpha 2-S$ (5'-CGA CGC CGA CCC CAA GGA CTA CC-3') and PLD $\alpha 2-A$ (5'-TCG CCG ACC CGA CGA TGA TGT AC-3'); PLD $\alpha 3-S$ (5'-CTG ACC CGA GGG ATT ACC TTA CC-3') and PLD $\alpha 3-A$ (5'-CAT GGA CCT CTG GTT GAT GTT GG-3'); PLD $\alpha 4-S$ (5'-CTC AAG GCG AAG AGG ATG GAC G-3') and PLD $\alpha 4-A$ (5'-TGG CCG ATC CCA CGA TGA TGT AC-3'); PLD $\alpha 5-S$ (5'-AGC GAC GCC GAC CCG AGG GAT TA-3') and PLD $\alpha 5-A$ (5'-GAT GTT GGC CGA CCC GAC GAT GA-3'); PLD $\beta 1$-S (5'-GGG TGC GTA TCA GCC ACA GTA T-3') and PLD $\beta 1$-A (5'-CAT TAT CAA CAA ATC GTT CCC A-3'); PLD $\beta 2$-S (5'-GAT CAA GTT CAG CCA ACA ATC CC-3') and PLDß2-A (5'-CAC AGT GAC ATC CTG TAC CCG TA-3');

PLD $\delta 1-S$ (5'-ATA CCG GCG TTT TAT GAT CTA TG-3') and PLD $\delta 1-A$ (5'-GAG GTC ATC AAC CAT CCC AAG A-3'); PLD $82-S$ (5'-TCG GAT CGG CCA ACA TCA ACC AG-3') and PLD $82-A$ (5'-TCC CTC ACC CGC CTC ACG CAC TC-3');

PLD $\varphi-S$ (5'-CCA CTG CAT GGG CAA GGT TGA GA-3') and PLD $\varphi$-A (5'-ATG AGG TTG CTG GTG CCG ATG TT-3');

Actin-1 (5'-GAA CTG GTA TGG TCAAGG CTG-3') and Actin-2 (5'-ACA CGG AGC TCG TTG TAG AAG-3').

\section{Isolation of PLD $\beta 1 c D N A$}

A rice EST clone (accession number C72286) that showed homology with PLD was found through an EST database search using Arabidopsis PLD (U84568) as bait. Based on the EST sequences, the specific primers PLD-1 (5'-GAT ACC CCG GCG TGC CC-3') and PLD-2 (5'-TGG TCG GCG TCC CTG ATC-3') were designed and used for isolating full-length cDNA from a library constructed of rice tiller material through PCR-based screening [43]. Plaque purified phage clones were converted to pBluescript SK derivatives using the helper phage ExAssist according to the supplier's instructions (Stratagene, USA). The cloned pPLD $\beta 1$, which contained the longest cDNA insert, was used for further analysis. DNA sequencing was performed by Genecore Company (Shanghai, China).

\section{RT-PCR and northern blot analysis of PLD $\beta 1$}

RT-PCR analyses were carried out to examine $P L D \beta 1$ expression in different tissues. In all, $5 \mu \mathrm{g}$ of total RNA, isolated from the roots, stem, leaves, spikes and immature seeds, was reverse transcribed and the resulting cDNAs were then used as templates for PCR amplification. The PLD-1 and PLD-2 primers were used. Rice actin1 was used as a positive internal control. A 2-week-old rice seedling was treated with $100 \mu \mathrm{M}$ IAA and ABA at $0,2,4,8$ and $12 \mathrm{~h}$ and used for RNA extraction. A 900-bp fragment of $P L D \beta 1$ served as a $[\alpha-32 \mathrm{P}] \mathrm{dCTP}-$ labeled hybridization probe.

\section{Transgenic approach and rice regeneration}

A 900-bp $P L D \beta 1$ fragment, digested from pPLD $\beta 1$ with SmaI and SalI, was subcloned to a p35S-1301 [44] vector precut with the same enzymes. The resulting binary vector, p35S-1301-antiPLD $\beta 1$ harboring $P L D \beta$ in an antisense orientation, was transferred to the Agrobacterium strain EHA105 and used for rice transformation. Rice transformation and regenerated resistant lines were confirmed using the method described in Liu et al. [44]. In total, 30 seeds from the confirmed $\mathrm{T} 1$ transgenic rice plants were germinated on hygromycinsupplemented selection medium for homozygous screening. The rice line in which all the seeds could germinate and grow normally was 
regarded as the homozygous line and was used for further analysis of the $\mathrm{T} 2$ generation.

Calculation of seed germination frequencies and observation of seedling growth

A total of 30 rice seeds from each homozygous transgenic line were germinated in medium supplemented with ABA (with concentrations at 10 or $20 \mu \mathrm{M}$ ) and in medium not supplemented with ABA. Seed germination frequencies were calculated after germination for 2, 3 and 4 days. All experiments were performed at least 3 times ( $\mathrm{n}>$ 30). To examine the effects of PA on seed germination and seedling growth, 50 rice seeds were germinated and grown under $50 \mu \mathrm{M}$ PA for 5 days. The primary root length and lateral root number were measured and statistically calculated. PA (1,2-diacyl-sn-glycero-3phosphate sodium salt, P9511) was first dissolved in chloroform and dried under a stream of helium. It was then dispersed into deionized water by sonication and added to the medium.

Expression analysis of GAmyb, $\alpha$-amylase, SAPK8 and SAPK10 by quantitative real-time RT-PCR

GAmyb, $\alpha$-amylase, SAPK8 and SAPK10 expression was analyzed by quantitative real-time RT-PCR. The seeds were imbibed in water and incubated for $24 \mathrm{~h}$, and then treated with PA $(50 \mu \mathrm{M})$ or ABA $(20 \mu \mathrm{M})$ for $24 \mathrm{~h}$. Total RNA was extracted, $2 \mu \mathrm{g}$ of total RNA was

Table 1 PLD gene family in Oryza sativa

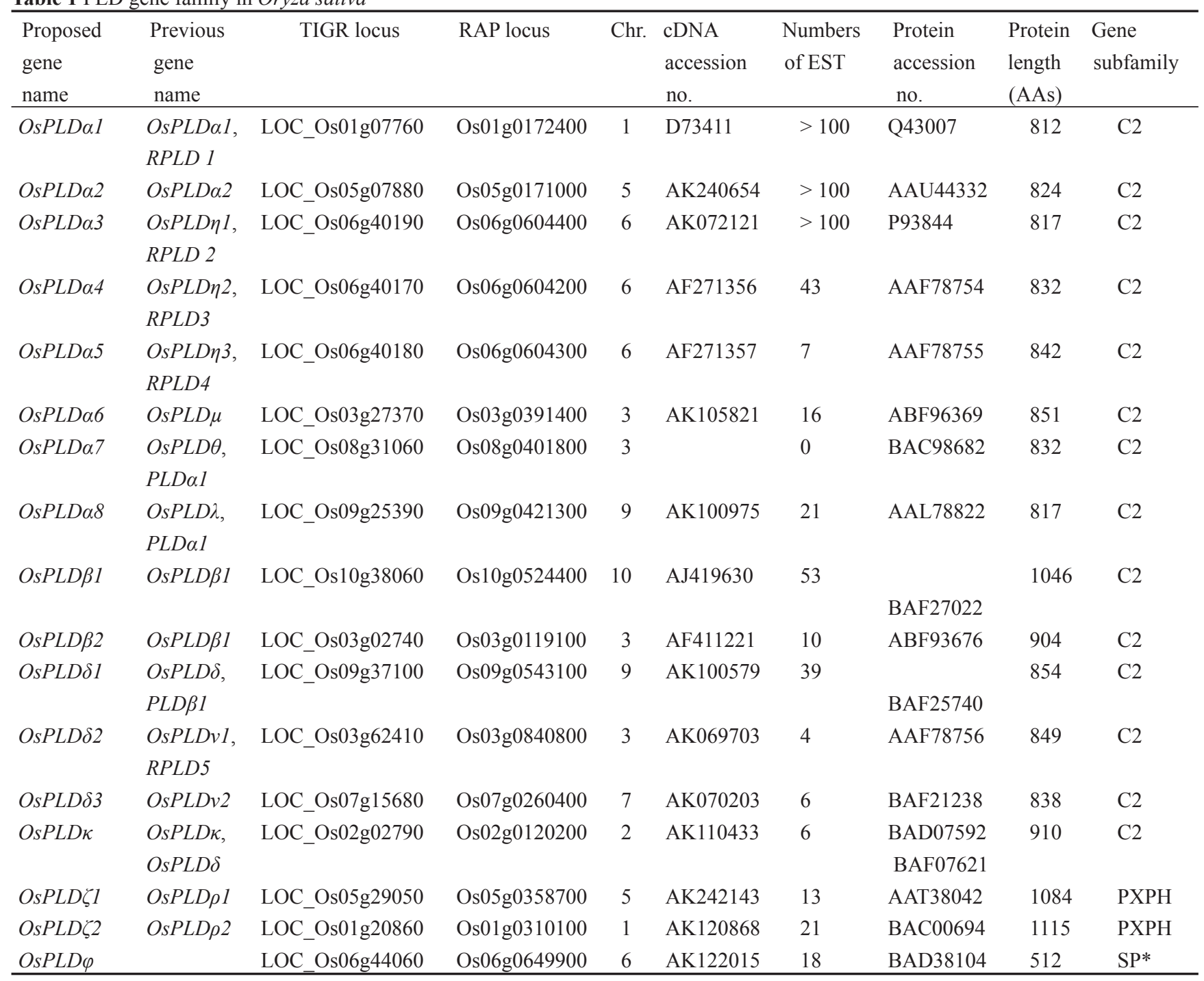

Note: Previous names (Genbank database or [39, 52]), RAP and TIGR locus numbers are listed. Please note that some members have more than one accession; those that have been annotated in the literature were selected with higher priority. The protein lengths and protein sequences come from the RAP database; except OsPLD $\alpha 6-7, \beta 2, \kappa$ and $\zeta 2$ (which come from the TIGR database). EST numbers are from a BLAST search of GenBank (up to April 12, 2007, E =0).*SP, Signal Peptide. 
reverse transcribed and qRT-PCR was performed as described above. Rice actin 1 was used as positive internal control. The primers used are listed as below:

GAmyb-1 (5'-CTG CGT TGC AGC CTA CTG AGT TA-3') and GAmyb-2 (5'-TAC ATG GCG TAC CGA CAG AAG AA-3'); $\alpha$-amylase-S (5'-CGG TGA TGG CTA CGCAAT CTG GG-3') and $\alpha$-amylase-A (5'-ATT CGG ATC GGA TAC AGC TCG TT-3');

SAPK8-S (5'-TAG TAT GAG CAG CCA GTA TGA GG-3') and SAPK8-A (5'-TCT TGT TGG TCG ATG ACT TAC AT-3');

SAPK10-S (5'-CTG TTC TTC ATT CGC AAC CAAAA-3') and SAPK10-A (5'-ATC CTC AAA AGG ATA TGC ACC AA-3').

\section{Results}

\section{Identification of 17 PLD genes in Oryza sativa}

To identify the rice PLD-coding genes, several approaches were used, including BLAST searches of databases (TIGR, Release 4; and RAP, Build 4.0) using Arabidopsis thaliana PLD genes as queries, keyword searches of the gene name "phospholipase" and ortholog searches using the conserved HKD domain (PF00614, PLD active site motif). The obtained results were combined and analyzed, resulting in the identification of 17 PLD members in rice. In particular, PLDא (Os02g02790), which was inaccurately annotated as a $\mathrm{C} 2$ protein previously, was shown to indeed encode a C2-PLD.

There are different annotations and gene names in the various databases, and disparate names have been used by different researchers. These names are disordered and lead to confusion for further studies. To make it clear, we have classified the 17 PLD members into three subgroups and have applied a uniform name according to protein sequence comparisons, peptide structures (conserved domains) and phylogenetic relationships with their respective orthologs in Arabidopsis (Table 1). Transcriptions of rice PLD genes are supported by isolated cDNAs or Expressed Sequence Tags (ESTs), except in the case of $P L D \alpha 7$.

\section{Structural organization and chromosomal distribution of rice PLDs}

Based on the predicted sequences, obtained cDNAs, and corresponding ESTs, the exon-intron structure of each PLD coding gene was determined (Figure 1). It has been reported that the exon-intron junctions of PLD genes are highly conserved within the individual subgroups in Arabidopsis $[10,39]$; however, these features are not well conserved in rice. In Arabidopsis C2-PLDs have 4 or 10 exons and all $P L D \alpha$ genes have 4 exons. However, in rice $P L D \alpha$ genes have random exon numbers, i.e. $4(P L D \alpha 6), 5(P L D \alpha 7)$ or 3 (PLD $\alpha 1,2,3,4,5,8)$ (Figure 1). There are 10 exons in rice $P L D \beta$ and $P L D \delta$, which are well conserved with those in Arabidopsis. $P L D \kappa$ is a special C2-PLD gene; it has 6 exons and no orthologous gene in Arabidopsis.

Regarding the genes encoding $P X P H-P L D s$, which consist of various numbers of exons, $P L D \zeta 1$ and $\zeta 2$ have 20

Table 2 Amino acid similarity between members of the PLD family in Oryza sativa

\begin{tabular}{|c|c|c|c|c|c|c|c|c|c|c|c|c|c|c|c|c|c|}
\hline $\mathrm{S} / \mathrm{I}$ & $\alpha 1$ & $\alpha 2$ & $\alpha 3$ & $\alpha 4$ & $\alpha 5$ & $\alpha 6$ & $\alpha 7$ & $\alpha 8$ & $\beta 1$ & $\beta 2$ & $\delta 1$ & $\delta 2$ & $\delta 3$ & $\kappa$ & $\zeta 1$ & $\zeta 2$ & $\varphi$ \\
\hline$\alpha 1$ & 1 & $86 / 79$ & $78 / 71$ & $73 / 66$ & $74 / 65$ & $71 / 64$ & $65 / 57$ & $61 / 52$ & $57 / 47$ & $56 / 48$ & $58 / 50$ & $58 / 49$ & $58 / 49$ & $54 / 46$ & $42 / 30$ & $41 / 29$ & $67 / 33$ \\
\hline$\alpha 2$ & & I & $75 / 68$ & $71 / 64$ & $72 / 64$ & $72 / 65$ & $65 / 58$ & $60 / 51$ & $57 / 46$ & $57 / 48$ & $57 / 49$ & $57 / 48$ & $56 / 46$ & $56 / 48$ & $40 / 30$ & $56 / 48$ & $26 / 26$ \\
\hline$\alpha 3$ & & & I & $78 / 73$ & $79 / 73$ & $70 / 63$ & $62 / 55$ & $61 / 53$ & $56 / 47$ & $57 / 49$ & $56 / 47$ & $55 / 47$ & $56 / 46$ & $54 / 46$ & $33 / 33$ & $40 / 30$ & $30 / 21$ \\
\hline$\alpha 4$ & & & & I & $87 / 85$ & $67 / 60$ & $60 / 53$ & $58 / 60$ & $56 / 47$ & $56 / 49$ & $56 / 48$ & $56 / 49$ & $56 / 48$ & $54 / 45$ & $42 / 30$ & $41 / 31$ & $32 / 23$ \\
\hline$\alpha 5$ & & & & & I & $67 / 60$ & $59 / 52$ & $57 / 49$ & $54 / 45$ & $55 / 47$ & $56 / 48$ & $55 / 46$ & $56 / 47$ & $54 / 46$ & $42 / 30$ & $39 / 30$ & $32 / 23$ \\
\hline$\alpha 6$ & & & & & & I & $69 / 62$ & $62 / 55$ & $56 / 44$ & $55 / 45$ & $58 / 49$ & $57 / 48$ & $57 / 43$ & $55 / 45$ & $34 / 26$ & $34 / 22$ & $26 / 21$ \\
\hline$\alpha 7$ & & & & & & & 1 & $55 / 47$ & $53 / 42$ & $53 / 42$ & $56 / 45$ & $54 / 45$ & $54 / 43$ & $51 / 42$ & $14 / 14$ & $14 / 14$ & $26 / 26$ \\
\hline$\alpha 8$ & & & & & & & & I & $53 / 44$ & $54 / 45$ & $54 / 46$ & $52 / 44$ & $52 / 43$ & $53 / 45$ & $34 / 24$ & $34 / 24$ & $37 / 26$ \\
\hline$\beta 1$ & & & & & & & & & / & $77 / 72$ & $65 / 56$ & $65 / 56$ & $64 / 55$ & $59 / 50$ & $40 / 30$ & $33 / 22$ & $40 / 20$ \\
\hline$\beta 2$ & & & & & & & & & & / & $64 / 55$ & $64 / 55$ & $64 / 56$ & $57 / 49$ & $38 / 28$ & $40 / 29$ & $34 / 23$ \\
\hline$\delta 1$ & & & & & & & & & & & I & $72 / 64$ & $72 / 62$ & $67 / 59$ & $54 / 46$ & $40 / 30$ & $80 / 60$ \\
\hline$\delta 2$ & & & & & & & & & & & & I & $82 / 76$ & $62 / 54$ & $38 / 29$ & $62 / 54$ & $30 / 20$ \\
\hline$\delta 3$ & & & & & & & & & & & & & I & $63 / 54$ & $40 / 30$ & $41 / 31$ & $33 / 0$ \\
\hline$\kappa$ & & & & & & & & & & & & & & / & $36 / 27$ & $38 / 29$ & $36 / 36$ \\
\hline$\zeta 1$ & & & & & & & & & & & & & & & I & $84 / 79$ & $20 / 20$ \\
\hline$\zeta 2$ & & & & & & & & & & & & & & & & 1 & $35 / 21$ \\
\hline
\end{tabular}

Note: The comparison was performed using Gap analysis from the GCG program and the results are presented as percentages. S, similarity; I, identity. 


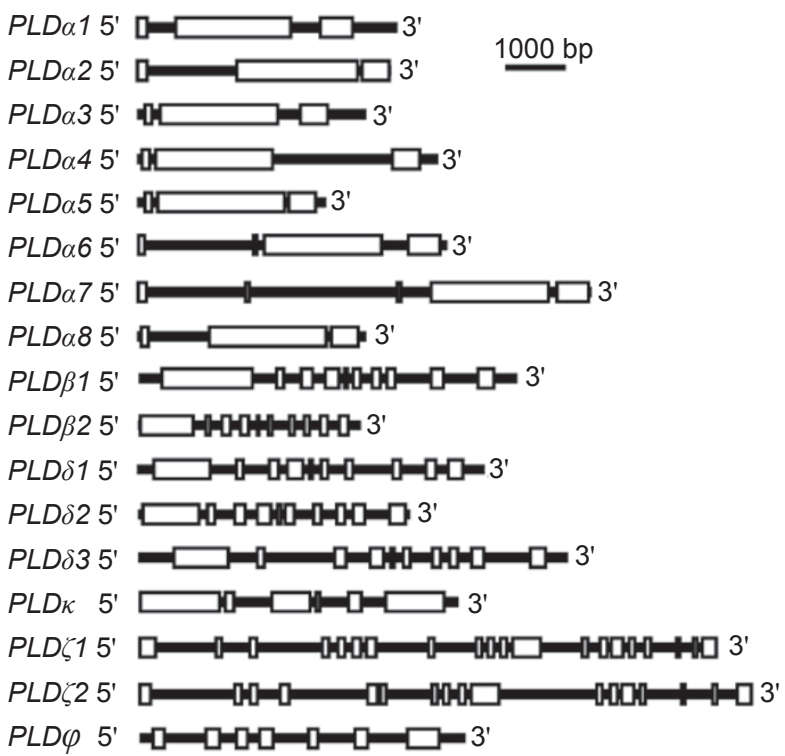

Figure 1 Exon-intron structures of rice $P L D$ genes. Exon-intron structures are determined by comparing cDNA (isolated or predicted) sequences with the relevant genomic sequences. Boxes represent exons and lines represent introns.

and 17 exons, respectively. This is similar to Arabidopsis, in which $P L D \zeta 1$ and $\zeta 2$ have 21 and 16 exons, respectively. Rice $P L D \varphi$, the unique member of the third subfamily (see following section), has 7 exons.

In general, rice $P L D$ genes are much larger than those in Arabidopsis and are distributed throughout 9 of the 12 chromosomes (they are not found on chromosomes 4, 11 and 12, Table 1). Interestingly, there is a tandem gene cluster located on chromosome 6 - PLD $\alpha 3-P L D \alpha 4-P L D \alpha 5$. These three genes share a very similar exon-intron structure, and are very similar at the protein level ( $>78 \%$ similarity, Table 2). This situation is analogous to the Arabidopsis $P L D \gamma 2-P L D \gamma 1-P L D \gamma 3$ cluster (sharing over $92 \%$ similarity), suggesting that the PLD family genes in Arabidopsis and rice may share conserved evolution patterns.

Domain structural analysis identified three subfamilies of rice PLDs

Based on their structural organization, rice PLDs can be classified into the C2-PLD, PXPH-PLD and SP-PLD subfamilies (Table 2; Figure 2). All rice PLDs contain two conserved HKD domains and highly conserved 'HKD' sequences, except for PLD $\alpha 7$, which lacks 'HK' in the second HKD domain (data not shown).

A conserved $\mathrm{C} 2$ domain is located at the $\mathrm{N}$-terminus of C2-PLD. There is a long region ( $200 \mathrm{Aa})$ located in front of the $\mathrm{C} 2$ domain of rice PLD $\beta 1$ (Figure 2) that is not present in other PLDs. Similar long regions are also present in Arabidopsis PLD $\beta 1$ (NP_565963, 274 Aa) and upload cotton (Gossypium hirsutum) PLD $\beta 1$ a (AAN05430, $274 \mathrm{Aa}$ ) and PLD $\beta 1 \mathrm{~b}$ (AAN05431, $352 \mathrm{Aa}$ ). However, the function of these long regions is still not known.

The PX and PH domains, located at the $\mathrm{N}$-terminus of PXPH-PLDs, are highly conserved in both plants and animals. The most evident difference is that rice PLD $\zeta 1$ has a shorter PH domain (25 Aa) than those of other PXPH-PLDs (rice PLD $22,93 \mathrm{Aa}$; Arabidopsis thaliana PLD $\zeta 1,91 \mathrm{Aa}$; Arabidopsis thaliana PLDל2, 114 Aa; Homo sapiens PLD1,

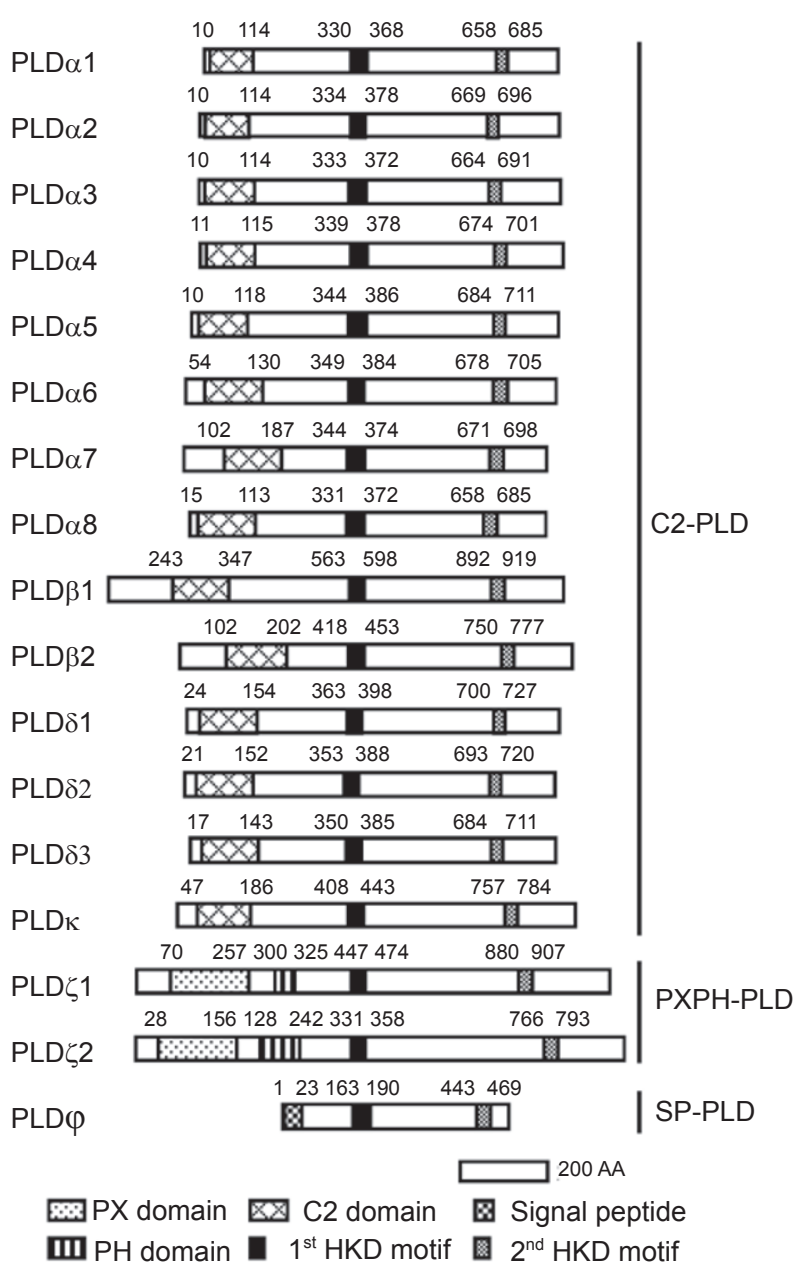

Figure 2 Peptide domain structures of rice PLD members. Schematic representations of the conserved domain and motif structures of rice PLDs are shown. Domain and motif structures were determined through searching the PLD protein sequences in PFAM and SMART. $\mathrm{C} 2$, protein kinase $\mathrm{C}$-conserved region 2; $\mathrm{PX}$, phox domain; $\mathrm{PH}$, pleckstrin homology domain; HKD, HxKxxxD, conserved catalytic region. 
104 Aa; and Homo sapiens PLD2, 108 Aa) (Figure 2).

A signal peptide is detected at the N-terminus of $\operatorname{PLD} \varphi$. As there is no other motif located in front of the first conserved HKD domain, PLD $\varphi$ is therefore designated as an SP-PLD. A similar type of PLD is also found in Caenorhabditis elegans (CAE72017, NP_504824), Dictyostelium discoideum (XP_637114) and mammals (Homo sapiens PLD3, AAH00553; PLD4, AAH15003), but has not been identified in other higher plants, including Arabidopsis thaliana, wheat or maize. There is a " $\mathrm{D}$ " to " $\mathrm{E}$ " substitution in the second HKD domain of mammalian SP-PLDs, while such a situation is not present in rice $\operatorname{PLD} \varphi$. This indicates that there is a long phylogenetic distance between rice and mammalian SP-PLDs. In addition, it is predicted that SPPLDs are secreted, and that their subcellular location is very similar to the secreted phospholipase-sPLA2. However, there is still no report on the physiological functions of SP-PLDs. Other domains, including PIP2-binding domain, FIYIENQYF domain and HYG, have also been detected in rice PLD members (data not shown).
A comparative analysis further showed that PLD members from the same subfamily share high protein-level similarity (Table 2). Of the C2-PLDs, 8 PLD $\alpha$ members share over 55\% similarity, 2 PLD $\beta$ members share $77 \%$ similarity and 3 PLD $\delta$ members share over $72 \%$ similarity. Two PLD $\zeta$ members share $84 \%$ similarity, but have less similarity $(<55 \%)$ with other PLDs. PLD $\varphi$ has low similarity with all other PLD members $(<40 \%)$, except PLD $\alpha 1$ (67\%) and PLD $\zeta 1$ (80\%).

Phylogenetic relationships of PLD genes in rice and Arabidopsis

To study the evolutionary relationships between different PLD members, GrowTree was constructed using a GCG program to analyze the phylogenetic relationships of PLDs from different species. There is a close phylogenetic relationship between PLD $\alpha$ members, as well as PLD $\beta$, PLD $\gamma$ and PLD $\delta$ members, in rice and Arabidopsis. However, there is a long phylogenetic distance between Arabidopsis PLDE, rice PLDк and other C2-PLDs (Figure 3A). Analysis

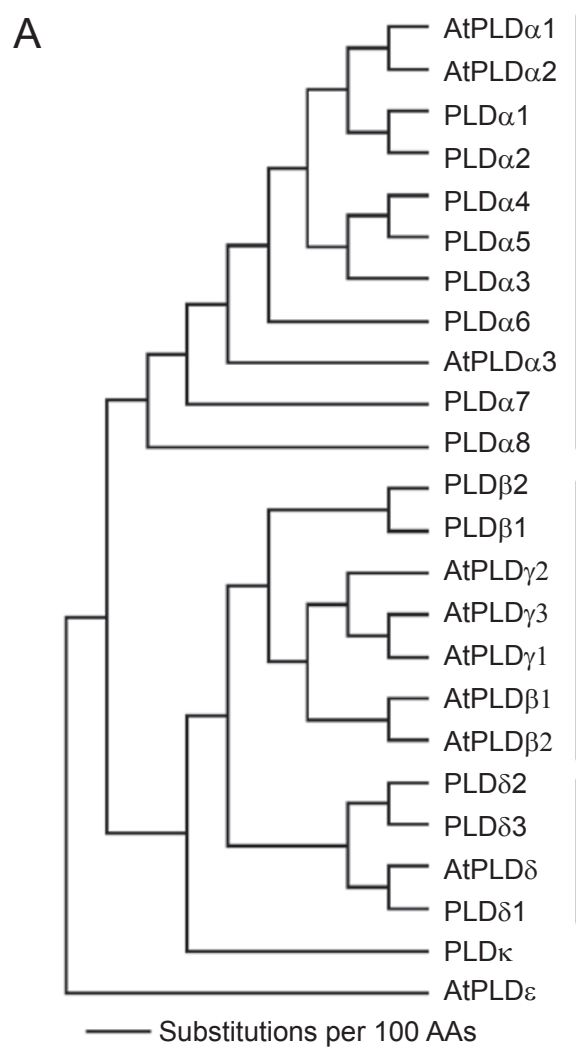

B

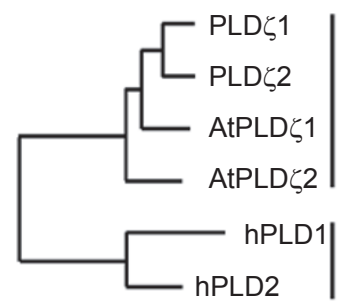

- Substitutions per $100 \mathrm{AAs}$

C

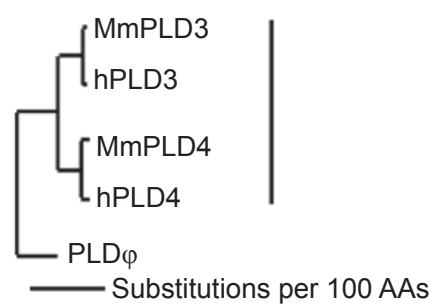

Figure 3 Phylogenetic relationships of PLD members in rice and Arabidopsis. GrowTrows were generated using the GCG program. The scale bars represent 100 amino acid substitutions per site. (A) A phylogenetic analysis of the relationship between C2-PLDs in rice and Arabidopsis. (B) A phylogenetic analysis of the relationship between PXPH-PLDs in rice, Arabidopsis and humans. (C) A phylogenetic analysis of the relationship between SP-PLDs in rice, humans and mice. 

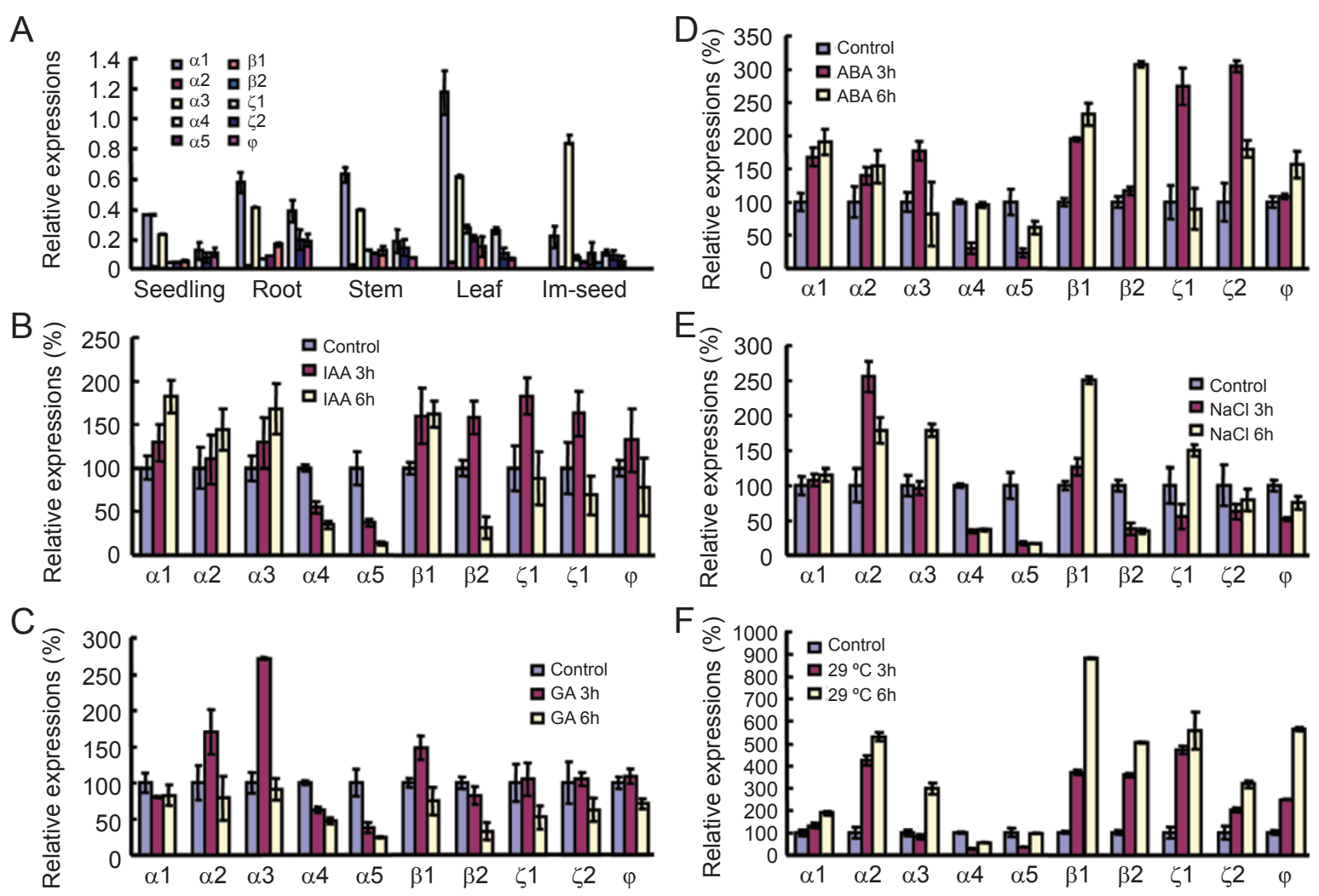

Figure 4 Expression of $P L D$ genes in various tissues and their response to environmental factors measured by quantitative real-time RT-PCR analysis. (A) Transcript levels of $P L D$ genes in various tissues of intact rice plants. (B-F) Relative expression of $P L D$ genes during treatment with IAA $(100 \mu \mathrm{M}$ for 3 or $6 \mathrm{~h}, \mathbf{B})$, GA $(100 \mu \mathrm{M}$ for 3 or $6 \mathrm{~h}, \mathbf{C}), \mathrm{ABA}(100 \mu \mathrm{M}$ for 3 or $6 \mathrm{~h}, \mathbf{D})$, salt $(250$ $\mathrm{mM} \mathrm{NaCl}$ for 3 or $6 \mathrm{~h}, \mathbf{E})$ or drought $\left(29^{\circ} \mathrm{C}\right.$ for 3 or $\left.6 \mathrm{~h}, \mathbf{F}\right)$.

of the PXPH-PLDs showed that rice PLD $\zeta$ members have a close phylogenetic relationship with their Arabidopsis orthologs, but there is a long phylogenetic distance to their mammalian orthologs (Figure 3B). Additionally, there is a long distance from PLD $\varphi$ to the mammalian PLDs (Figure $3 \mathrm{C})$.

PLDs are expressed in various tissues and are involved in multiple cellular responses to hormones and environmental stimuli

Quantitative real-time RT-PCR analysis was performed to examine the expression patterns of PLD genes. As shown in Figure 4A, most $P L D$ genes are expressed in a variety of tissues, including seedlings, roots, stems, leaves and immature seeds, although some of them have lower expression levels (expression levels of $P L D \alpha 6-8, \delta 1-3$ and $\kappa$ are too low to be detected by qRT-PCR).

Many rice $P L D$ genes are induced by plant hormones (IAA, GA, ABA) or abiotic stress (salt and drought stress) (Figure 4B-4F). PLD $\alpha 1$ is induced by IAA, ABA and drought, and is suppressed by GA; $P L D \alpha 2$ is highly induced by salt and drought; $P L D \alpha 3$ is induced by most plant hormones and stress stimuli; while $P L D \alpha 4$ and $P L D \alpha 5$ are suppressed by most plant hormones and stress stimuli. $P L D \alpha 1$ is induced by most plant hormones and stress stimuli; and $P L D \beta 1, P L D \zeta 1$ and $P L D \zeta 2$ are induced by IAA, ABA and drought, and suppressed by salt stress. These differential expression patterns reveal the specific and distinct roles of different $P L D$ genes in hormone effects and stress responses.

Isolation of PLD $\beta 1$, which encodes a C2-PLD and is induced by $A B A$

Of the 17 rice $P L D s$, only 5 have been cloned individually, and none of them has been functionally characterized yet. To elucidate the physiological function of PLDs in rice, cDNA encoding $P L D \beta 1$ was isolated. In short, a rice EST (C72286) sharing high similarity with $P L D \beta 1$ at amino acid positions $700-818$ was identified by searching the dbEST database with the Arabidopsis PLD 1 (U84568) 
coding sequence. Specific primers were then designed and used for cDNA library screening via PCR-based methods [43]. The resulting cDNA clone (Accession No. AJ419630), with a length of $3180 \mathrm{bp}$ and an open reading frame between nucleotides 478 (ATG) and 3015 (TGA), encodes a protein containing $845 \mathrm{AA}$. On comparing the fragment with the recently released full-length rice cDNA sequences, we found that this fragment is a partial cDNA of $P L D \beta 1$ (longer cDNA clones were isolated: AK073012 and AK121075 [45]).

Northern blot analyses indicated relatively low expression of $P L D \beta 1$ in various tissues, and further RT-PCR analysis showed that $P L D \beta 1$ is expressed in root, stem, leaf, spike and immature seeds (Figure $5 \mathrm{~A}$ ). In addition, northern blot analyses confirmed that $P L D \beta 1$ is rapidly induced by IAA and ABA (Figure 5B).
Deficiency of $P L D \beta 1$ results in repressed responses to exogenous $A B A$

A transgenic approach was used to study the physiological function of $P L D \beta 1$. A construct $\mathrm{pA}-\mathrm{PLD}$ harboring $P L D \beta 1$ in an antisense orientation was transformed into rice using an immature embryo, and more than 10 resistant plants were obtained after initial screening. Harvested $\mathrm{T} 1$ seeds were germinated on medium supplemented with hygromycin to confirm the resistance. Single-copy integration of T-DNA was confirmed by the germination frequency (a 3:1 ratio) on selective medium, and further RT-PCR analysis confirmed the suppressed expression of $P L D \beta 1$ in transgenic plants (Figure 5C). The harvested $\mathrm{T} 2$ seeds were then used to identify homozygous lines for further study.

Under normal growth conditions, there is no evident
A

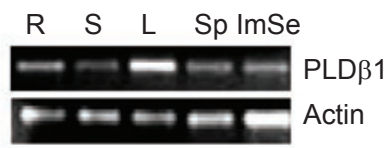

B

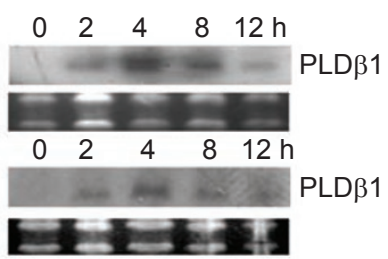

C

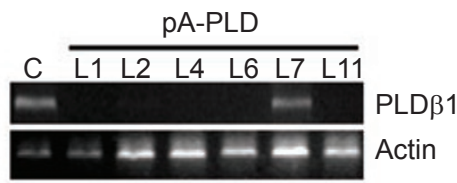

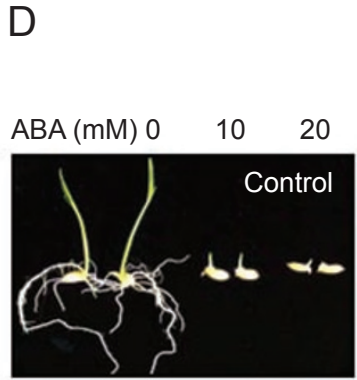
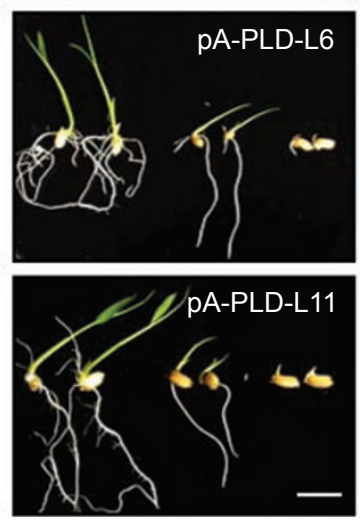
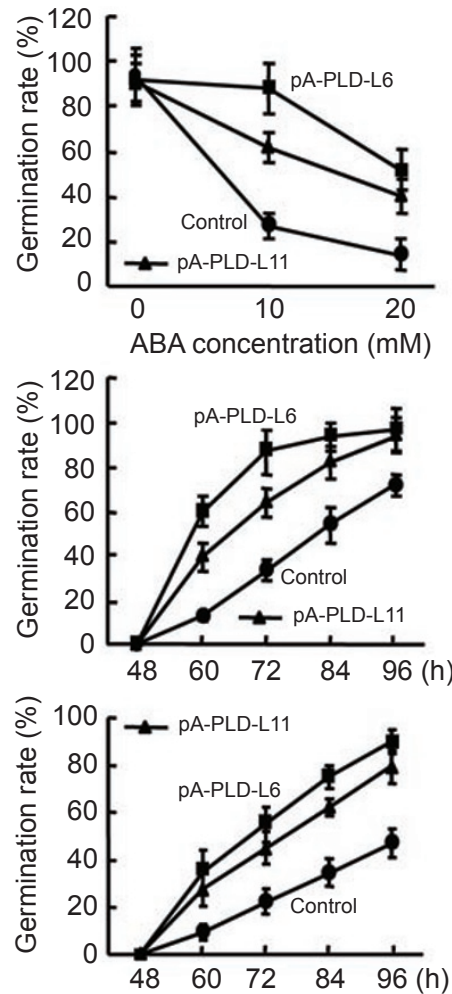

Figure $5 P L D \beta 1$ deficiency results in reduced sensitivity to exogenous ABA. (A) Transcript levels of $P L D \beta 1$ in various tissues of intact rice plants, revealed through RT-PCR. R, root; S, stem; L, leaf; Sp, spike; and ImSe, immature seeds. (B) Northern blot analysis showed that both IAA (upper panel) and ABA (lower panel) stimulate $P L D \beta 1$ expression. Rice shoots (germinated for 10 days) were treated with $100 \mu \mathrm{M}$ IAA for $0,2,4,8$ and $12 \mathrm{~h}$, or $100 \mu \mathrm{M}$ ABA for $0,2,4,8$ and $12 \mathrm{~h}$. (C) Repressed expression of $P L D \beta 1$ in transgenic plants harboring pA-PLD. Total RNA was extracted from independent 14-day-old transgenic plants (lines 1, 2, 4, 6, 7, 11) and $P L D \beta 1$ transcripts were analyzed through RT-PCR. The Actin gene was used as an internal positive control. (D) Suppressed expression of $P L D \beta 1$ resulted in decreased ABA inhibition of seed germination and seedling growth. Seedlings were grown for 5 days under different concentrations of $\operatorname{ABA}(0,10$ or $20 \mu \mathrm{M}$; in the left panels the scale bar represents $1 \mathrm{~cm})$. Germination ratios were measured and statistically calculated after treatment with $\mathrm{ABA}(0,10$ or $20 \mu \mathrm{M})$ for $72 \mathrm{~h}$ (right panel, upper) or at $10 \mu \mathrm{M}$ (right panel, middle) or $20 \mu \mathrm{M}$ (right panel, bottom) for different durations (48, 60, 72, 84 or $96 \mathrm{~h}$ ). Error bars represent the SE (n $>30$ ). 

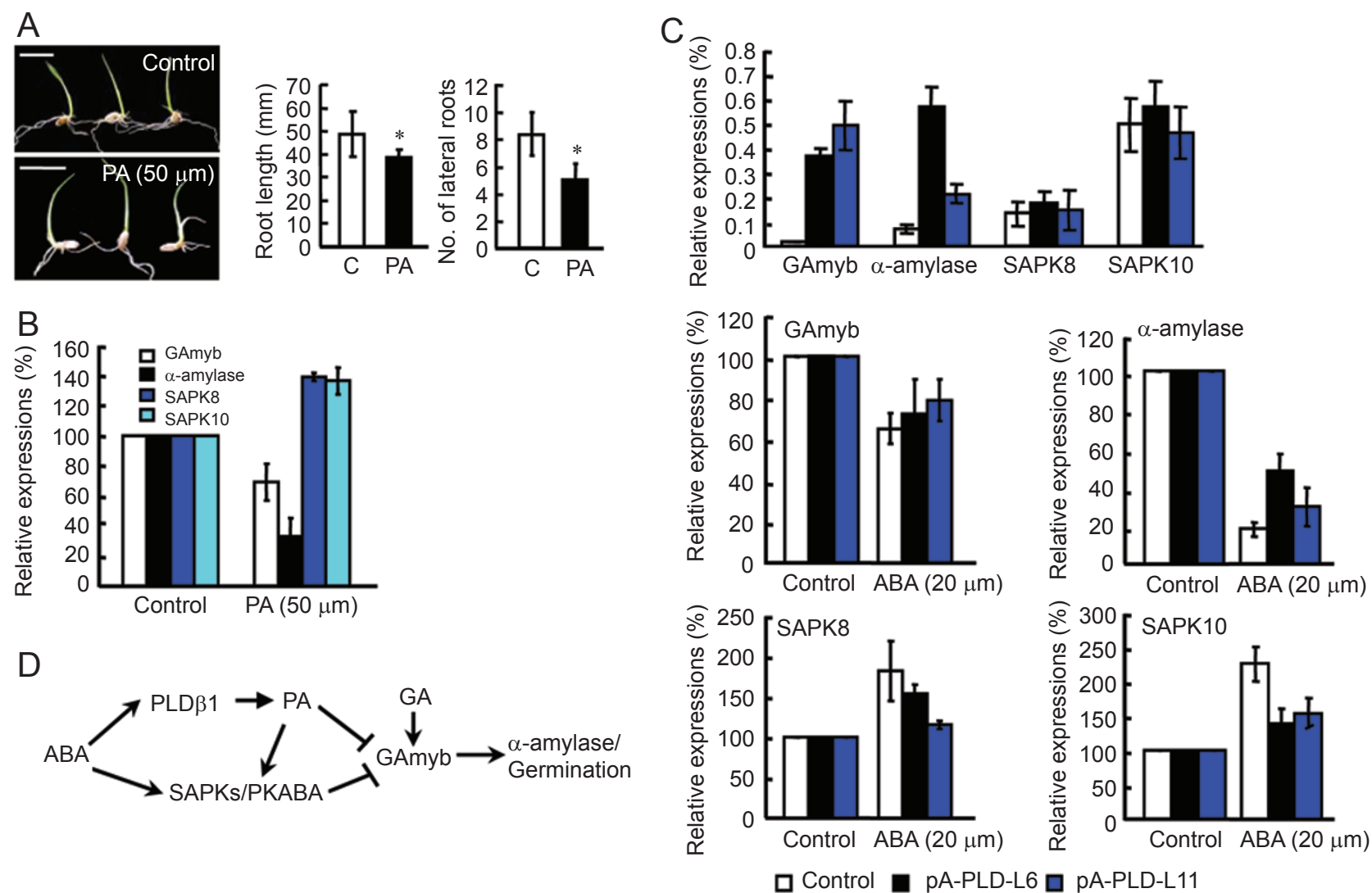

Figure $6 P L D \beta 1$ is involved in seed germination and seedling growth through the regulation of $S A P K / P K A B A$ and $G A m y b$ expression. (A) PA represses rice seedling growth. Seedlings were grown on medium supplemented with $50 \mu \mathrm{M}$ of PA and medium not supplemented with PA for 5 days (left). Primary root length (middle panel) and lateral root numbers (right panel) of 14-day-old seedlings were measured and statistically calculated $(P<0.01)$. Scale bar represents $1 \mathrm{~cm}$. (B) PA $(50 \mu \mathrm{M}$ for $24 \mathrm{~h})$ inhibits $G A m y b$ and $\alpha$-amylase expression, and stimulates $S A P K 8$ and SAPK10 expression. (C) The relative expression of GAmyb, $\alpha$-amylase, SAPK 8 and $S A P K 10$ in early seed germination without or with treatment of ABA in rice. $P L D \beta 1$ deficiency results in stimulated expression of $G A m y b$ and $\alpha$-amylase, while the expression of $S A P K 8$ and $S A P K 10$ is not altered (upper panel). Under treatment with ABA (20 $\mu \mathrm{M})$, ABA-inhibited expression of GAmy $b$ and $\alpha$-amylase, and ABA-stimulated expression of $S A P K 8$ and $S A P K 10$ are much repressed (middle and lower panels). (D) A hypothetical model of the roles of $P L D \beta 1$ in ABA-regulated seed germination.

difference between control and transgenic plants. As $P L D \beta 1$ is induced by ABA, and PLD has been shown to be involved in the ABA response in barely aleuronic cells [21] and Arabidopsis seed germination [23, 37], we focused on $P L D \beta 1$ effects in ABA responses. Phenotypic and statistical analysis of seed germination reveals a repressed sensitivity to exogenous ABA in $P L D \beta 1$-deficient plants (Figure 5D). Seed germination and seedling growth of wild-type plants are heavily inhibited by exogenous ABA, while the inhibitory effects are much reduced in $P L D \beta 1$ -deficient plants.

PLD $\beta 1$ deficiency modulates the expression of SAPK, GAmyb and $\alpha$-amylase

To further study the role of PLD $\beta 1$ and PA in ABA signaling transduction during seed germination, the effects of PA on seed germination and seedling growth were examined. In Arabidopsis, the amount of PA reaches a maximum at $12 \mathrm{~h}$ and decreases after $96 \mathrm{~h}$ during the early stages of seed germination. In addition, a deficiency of LPP results in a significant increase of PA and hypersensitive responses to ABA during seed germination [23]. As shown in Figure 6A, long-term treatment with PA $(50 \mu \mathrm{M}$ for 5 days) inhibited seedling growth, especially primary root elongation and lateral root formation. This suggests a negative regulatory role for PA in seedling growth, which is consistent with the previous report that PA application will mimic the ABA effects [21].

Studies using barley aleurone showed that PLD and its product PA are involved in the ABA-suppressed and 
GA-stimulated expression of $G A m y b$ and $\alpha$-amylase genes through regulating PKABA1, an ABA-activated SNF1 (sucrose nonfermenting protein kinases) type of protein kinase in barley [46-49]. In addition, Arabidopsis SnRK2 (SNF1related protein kinase 2) is orthologous to PKABA1 [50]. SnRK2.6/OST1/SRK2E, the best characterized member of SnRK2 family, was activated by ABA and has essential roles in ABA signal transduction [51].

We thus tried to detect whether a similar regulatory mechanism operates during the process of rice seed germination. It has been shown that rice SAPK family genes are orthologous to PKABA1, and analysis of protein sequences and domain structures shows that rice SAPK8 and SAPK10 are most orthologous to PKABA1 and SnRK2.6/ OST1/SRK2E, and, more importantly, that both of them are activated by ABA [50].

First, we studied the effects of PLD $\beta 1$ and PA on the expression of SAPK8 and SAPK10, GAmyb and $\alpha$-amylase. As shown in Figure $6 \mathrm{~B}$, expression of $S A P K 8$ and SAPK10 is stimulated by PA, while expression of GAmyb and $\alpha$-amylase is clearly inhibited during treatment with $\mathrm{PA}$, indicating that PA has similar effects as ABA on the expression of these genes. With PLD $\beta 1$ deficiency, the expression of $G A m y b$ and $\alpha$-amylase is clearly enhanced during early seed germination in rice (Figure 6C, upper panel), which is consistent with the observation that the expression of GAmyb and $\alpha$-amylase is inhibited during treatment with PA. Further analysis shows that treatment with ABA $(20 \mu \mathrm{M}$ for $24 \mathrm{~h})$ seriously suppresses the expression of $G A m y b$ and $\alpha$-amylase during seed germination in rice. However, the inhibitory effects are much reduced with $P L D \beta 1$ deficiency (Figure $6 \mathrm{C}$, middle panel), which suggests the involvement of $P L D \beta 1$ in mediating the function of ABA. In addition, although expression of SAPK8 and SAPK10 was not significantly altered with $P L D \beta 1$ deficiency (Figure 6C, upper panel), the clearly enhanced expression of $S A P K 8$ and $S A P K 10$ by ABA was much reduced, similar to the untreated control (Figure $6 \mathrm{C}$, lower panel), indicating a specific role for $P L D \beta 1$ in ABA-induced $S A P K$ expression. This suggests that $P L D \beta 1$ is positively involved in the ABA-mediated inhibition of GAmyb and $\alpha$-amylase expression, partially through $S A P K$ regulation (Figure 6D).

\section{Discussion}

Although there are 17 PLDs in rice, only 6 of them have been isolated and studied for their expression patterns and subcellular locations [52], and only $P L D \beta 1$ has been physiologically characterized. The specific expression patterns of $P L D$ genes may hint at their unique roles in plant growth and development.

\section{PLD family in Oryza sativa}

Through a reiterative database search of public databases, 17 PLD coding genes were identified in rice, and they were shown to share high sequence identity to their orthologs in Arabidopsis. The close phylogenetic relationship between rice PLDs and their orthologs in Arabidopsis suggests that the evolution of PLDs in different species is well conserved. Further exon-intron organizational studies indicate that the structures of rice PLD genes are more complex than their orthologs in Arabidopsis. Protein domain structure analysis reveals that all PLD members in rice, except PLD $\alpha 7$, contain two conserved HKD domains. PLD $\alpha 7$ has a mutation in the second HKD domain. Based on the domain structures, rice PLDs can be classified into three subfamilies, which include the C2-PLDs (14 members), the PXPH-PLDs (2 members) and the SP-PLD (1 member). This new type of PLD, SP-PLD, has a signal peptide rather than a $\mathrm{C} 2$ domain or PXPH domain at the $\mathrm{N}$-terminus. It is predicted that it can be secreted out of the cell, and it is the first identified secreted PLD in higher plants. The specific localization of SP-PLD, just like sPLA2 in higher plants, suggests its specific physiological function in plant growth and developmental processes, such as plant defense. Interestingly, this type of PLD was identified in most animals, but in higher plants it was identified only in rice, revealing its unique role in PLD evolution.

Apart from $P L D \alpha 7$, rice $P L D$ genes have been shown to be expressed in various tissues based on the support of isolated cDNAs or ESTs. There is no corresponding EST or cDNA support for $P L D \alpha 7$, indicating that PLD $\alpha 7$ maybe transcribed at a very low level or under specific conditions. Furthermore, $P L D \alpha 7$ might be an artificial gene, because there is no EST or cDNA support, and a 'D' to ' $E$ ' substitution in the second HKD domain might result in loss of protein function. Further quantitative real-time RT-PCR analyses confirm the expression of rice $P L D$ genes in various tissues, although some of them are expressed at lower levels. Most PLD genes are induced by hormones (IAA, GA, ABA) or stress stimuli (salt and drought), indicating the possible involvement of PLDs in hormone effects and stress responses. In Arabidopsis, PLDs have been shown to be involved in multiple processes; for example, PLD $\alpha 1$ is involved in the signal transduction of ABA, GA, and ethylene, and in senescence, water loss and freezing tolerance; PLD $\delta 1$ is a positive regulator of stress response, and also acts as a bridge between the plasma membrane and microtubule $[13,16]$; PLD $\zeta 2$ is involved in phosphorusdeficiency-induced root elongation and digalactosyldiacylglycerol accumulation [41, 42], and is required for auxin response [26]. In rice, the distinct expression patterns of different PLD members in the presence of plant hormones and in stress responses reveal the multiple and specific roles 
of PLDs. This is consistent with previous studies. Previous studies have indicated that rice PLDs have an overlapping distribution but also have distinct expression patterns [52], which are induced by hydrogen peroxide [53] and are involved in elicitor-induced phytoalexin accumulation [52], thus indicating the specific and important roles of PLDs in plant growth and development.

PLD 1 mediates $A B A$ response and is involved in seed germination

Seed germination is a complex process controlled by many factors including light, temperature and plant hormones. GA is believed to promote seed germination, while ABA induces seed dormancy in maturing embryos and prevents seed germination $[55,56]$. Previous studies have shown that PLDs and PA are involved in ABA- and GA-regulated seed germination. PLD activity and the amount of PA are increased during seed germination and the seedling stage $[23,36,57]$. In addition, PLD is one of the target proteins of ABA, and supplementation of PA into the endosperm of barley resulted in ABA-like inhibition of the GA response [21], indicating that PLD may involve or mediate $\mathrm{ABA}$ signal transduction during seed development.

Our studies show that rice $P L D \beta 1$ positively regulates the ABA response in seed germination. $P L D \beta 1$ deficiency results in decreased sensitivity to ABA during seed germination (Figure 5) and reduced tolerance to salt stress (Supplementary information, Figure S1), in a way similar to Arabidopsis PLD $\alpha 1$-deficient plants, which have decreased stomatal closure induced by ABA and reduced tolerance to drought stress [37]. In addition, PA is able to mimic ABA function in activating the expression of $S A P K 8$ and SAPK10, suppressing the expression of GAmyb and $\alpha$-amylase, and inhibiting seedling growth (Figure 6), providing further evidence that PLD regulates the ABA response through its product $\mathrm{PA}$, which is in agreement with previous investigations [21]. Although $P L D \beta 1$ plays a positive role in the ABA response during seed germination, at present we cannot exclude the possibility that other PLD members also participate in the ABA response. Indeed, besides $P L D \beta 1$, several other rice $P L D$ genes, including $P L D \alpha 1, \alpha 3, \beta 2$ and $\delta 2$, can also be induced by ABA(Figure 4 ), which suggests that they might be involved in the ABA response as well.

In barley aleurone cells, PLD and PA are involved in ABA-suppressed and GA-stimulated GAmyb and $\alpha$-amylase gene expression through the regulation of PKABA1 expression [46-48]. Expression pattern analysis shows that $S A P K 8$ and SAPK10, which are rice orthologs of PKABA1, are induced by ABA, and that this type of induction is repressed with $P L D \beta 1$ deficiency. This indicates that $P L D \beta 1$ is involved in the regulation of ABA-induced $S A P K$ expression. However, the unaltered expression of $\mathrm{S} A P K 8$ and $S A P K 10$ in $P L D \beta 1$-deficient rice in the absence of ABA suggests the presence of other mechanisms for controlling $S A P K 8$ and SAPK10 expression. In addition, reduced inhibition of $G A m y b$ and $\alpha$-amylase expression by ABA in $P L D \beta 1$-deficient rice further confirms the negative roles of $P L D \beta 1$, as well as PA, in seed germination (Figure 6D).

Recent studies have shown that Arabidopsis SnRK2.10, an ortholog of rice SAPK and barely PKABA1 [50], is the direct target protein of PA [58], revealing the possibility that SAPK may be the direct target protein of PA in rice. These results suggest that, during the process of seed germination, $P L D \beta 1$-derived PA may inhibit the expression of $G A m y b$ and $\alpha$-amylase directly or indirectly through activating SAPK functions, and thus may be negatively involved in seed germination (Figure 6D). In addition, it has been shown that ABA-induced activation of SnRK2.6/ OST1/SRK2E, the closest Arabidopsis ortholog of SAPK8 and SAPK10, is inhibited by ABI1 through its binding to the conserved domain II at the N-terminus of SRK2E [57]. Furthermore, as ABI1 activity is inhibited by PA [22], we may thus speculate that PLD and PA may activate SRK2E functions through inhibiting ABI1 activities. The presence of the conserved domain II at the SAPK8 and SAPK10 $\mathrm{N}$-termini further supports this possibility; however, this needs to be further examined.

Expression of $P L D \beta 1$ is regulated not only by ABA but also by auxin, GA, salt and drought. The fact that $P L D \beta 1$-deficient plants showed increased sensitivity to salt (Supplementary information, Figure S1) and treatment with GA (data not shown) suggests that $P L D \beta 1$ might be involved in the effects of multiple plant hormones and in stress tolerance.

\section{Acknowledgments}

This work was supported by the State Key Project of Basic Research (2005CB120803) and the National Natural Science Foundation of China (30425029, 30421001). We thank Ms Shu-Ping Xu (Institute of Plant Physiology and Ecology, Shanghai Institutes for Biological Sciences, Chinese Academy of Sciences) for help on rice transformation.

\section{Accession numbers}

Sequence data from this article can be found on the GenBank website. Accession numbers for rice PLDs are listed in Table 1. For Arabidopsis PLDs, please refer to Qin and Wang (2002) [10]. Other sequences are listed as follows: hPLD1 (AAH68976), hPLD2 (AAB96655), hPLD3 (AAH00553), hPLD4 (AAH15003), MmPLD3 (AAH76586), MmPLD4 (AAH58565), GAmyb (OsGAMYB, X98355), $\alpha$-amylase (OsALAM, X16509), SAPK8 (AB125309) and SAPK10 (AB125311) 


\section{References}

1 Morris AJ, Engebrecht J, Frohman MA. Structure and regulation of phospholipase D. Trends Pharmacol Sci 1996; 17:182-185.

2 Hanahan DJ, Chaikoff IL. A new phospholipid splitting enzyme specific for the ester linkage between the nitrogenous base and the phosphoric acid grouping. J Biol Chem 1947; 169:699-705.

3 Wang X, Xu L, Zheng L. Cloning and expression of phosphatidylcholine-hydrolyzing phospholipase D from Ricinus communis L. J Biol Chem 1994; 269:20312-20317.

4 Qin W, Pappan K, Wang X. Molecular heterogeneity of phospholipase D (PLD). Cloning of PLDgamma and regulation of plant PLDgamma, -beta, and -alpha by polyphosphoinositides and calcium. J Biol Chem 1997; 272:28267-28273.

5 Ueki J, Morioka S, Komari T, Kumashiro T. Purification and characterization of phospholipase D (PLD) from (Oryza sativa L.) and cloning of cDNA for PLD from rice and maize (Zea mays L.). Plant Cell Physiol 1995; 36:903-914.

6 Morioka S, Ueki J, Komari T. Characterization of two distinctive genomic clones (accession nos. AB001919 and AB001920) for phospholipase D from rice (PGR 97-076). Plant Physiol 1997; 114:396.

7 Lein W, Saalbach G. Cloning and direct G-protein regulation of phospholipase D from tobacco. Biochim Biophys Acta 2001; 1530:172-183.

8 Whitaker BD, Smith DL, Green KC. Cloning, characterization and functional expression of a phospholipase Dalpha cDNA from tomato fruit. Physiol Plant 2001; 112:87-94.

9 Wang X. Multiple forms of phospholipase D in plants: the gene family, catalytic and regulatory properties, and cellular functions. Prog Lipid Res 2000; 39:109-149.

10 Qin C, Wang X. The Arabidopsis phospholipase D family characterization of a calcium-independent and phosphatidylcholineselective PLD $\zeta 1$ with distinct regulatory domains. Plant Physiol 2002; 128:1057-1068.

11 Munnik T, Irvine RF, Musgrave A. Phospholipid signalling in plants. Biochim Biophys Acta 1998; 1389:222-272.

12 Wang X. Phospholipase D in hormonal and stress signaling. Curr Opin Plant Biol 2002; 5:408-414.

13 Wang X. Regulatory functions of phospholipase D and phosphatidic acid in plant growth, development, and stress responses. Plant Physiol 2005; 139:566-573.

14 Gardiner J, Collings DA, Harper JD, Marc J. The effects of the phospholipase D-antagonist 1-butanol on seedling development and microtubule organization in Arabidopsis. Plant Cell Physiol 2003; 44:687-696.

15 Ohashi Y, Oka A, Rodrigues-Pousada R, et al. Modulation of phospholipid signaling by GLABRA2 in root-hair pattern formation. Science 2003; 300:1427-1430.

16 Monteiro D, Liu Q, Lisboa S, Scherer GE, Quader H, Malho R. Phosphoinositides and phosphatidic acid regulate pollen tube growth and reorientation through modulation of $\left[\mathrm{Ca}^{2+}\right] \mathrm{c}$ and membrane secretion. J Exp Bot 2005; 56:1665-1674.

17 Dhonukshe P, Laxalt AM, Goedhart J, Gadella TWJ, Munnik T. Phospholipase D activation correlates with microtubule reorganization in living plant cells. Plant Cell 2003; 15:2666-2679.

18 Motes CM, Pechter P, Yoo CM, Wang YS, Chapman KD, Blancaflor EB. Differential effects of two phospholipase D inhibitors, 1-butanol and $\mathrm{N}$-acylethanolamine, on in vivo cytoskeletal or- ganization and Arabidopsis seedling growth. Protoplasma 2005; 226: $109-123$.

19 Hirase A, Hamada T, Itoh TJ, Shimmen T, Sonobe S. n-Butanol induces depolymerization of microtubules in vivo and in vitro. Plant Cell Physiol 2006; 47:1004-1009.

20 Fan L, Zheng S, Wang X. Antisense suspension of PLD alpha retards abscisic acid and ethylene-promoted senescence of postharvest Arabidopsis. Plant Cell 1997; 9:2183-2196.

21 Ritchie S, Gilroy S. Abscisic acid signal transduction in the barley aleurone is mediated by phospholipase D activity. Proc Natl Acad Sci USA 1998; 95:2697-2702.

22 Zhang W, Qin C, Zhao J, Wang X. Phospholipase Dá1-derived phosphatidic acid interacts with ABI1 phosphatase 2C and regulates abscisic acid signaling. Proc Natl Acad Sci USA 2004; 101:9508-9513.

23 Katagiri T, Ishiyama K, Kato T, Tabata S, Kobayashi M, Shinozaki K. An important role of phosphatidic acid in ABA signaling during germination in Arabidopsis thaliana. Plant J 2005; 43:107-117.

24 Romanov GA, Kieber JJ, Schmulling T. A rapid cytokinin response assay in Arabidopsis indicates a role for phospholipase D in cytokinin signalling. FEBS Lett 2002; 515:39-43.

25 Wang C, Zien CA, Afitlhile M, Welti R, Hildebrand DF, Wang X. Involvement of phospholipase D in wound-induced accumulation of jasmonic acid in Arabidopsis. Plant Cell 2000; 12:22372246.

$26 \mathrm{Li} \mathrm{G}$, Xue HW. Arabidopsis $P L D \zeta 2$ regulates vesicle trafficking and is required for auxin response. Plant Cell 2007; 19:281295.

27 Finkelstein RR, Gampala SS, Rock CD. Abscisic acid signaling in seeds and seedlings. Plant Cell 2002; 14 (Suppl):S15-S45.

28 Razem FA, El-Kereamy A, Abrams SR, Hill RD. The RNAbinding protein FCA is an abscisic acid receptor. Nature 2006; 439:290-294.

29 Shen YY, Wang XF, Wu FQ, et al. The Mg-chelatase H subunit is an abscisic acid receptor. Nature 2006; 443:823-826.

30 Sheen J. Mutational analysis of protein phosphatase $2 \mathrm{C}$ involved in abscisic acid signal transduction in higher plants. Proc Natl Acad Sci USA 1998; 95:975-980.

31 Gosti F, Beaudoin N, Serizet C, Webb A, Vartanian N, Giraudat J. $\mathrm{ABI} 1$ protein phosphatase $2 \mathrm{C}$ is a negative regulator of abscisic acid signaling. Plant Cell 1999; 11:1897-1909.

32 Wang XQ, Ullah H, Jones AM, Assmann SM. G protein regulation of ion channels and abscisic acid signaling in Arabidopsis guard cells. Science 2001; 292:2070-2072.

33 Jacob T, Ritchie S, Assmann SM, Gilroy S. Abscisic acid signal transduction in guard cells is mediated by phospholipase D activity. Proc Natl Acad Sci USA 1999; 96:12192-12197.

34 Mishra G, Zhang W, Deng F, Zhao J, Wang X. A bifurcating pathway directs abscisic acid effects on stomatal closure and opening in Arabidopsis. Science 2006; 312:264-266.

35 Zalejski C, Zhang Z, Quettier AL, et al. Diacylglycerol pyrophosphate is a second messenger of abscisic acid signaling in Arabidopsis thaliana suspension cells. Plant J 2005; 42:145152.

36 Ritchie S, Gilroy S. Abscisic acid stimulation of phospholipase $\mathrm{D}$ in the barley aleurone is G-protein-mediated and localized to the plasma membrane. Plant Physiol 2000; 124:693-702.

37 Sang Y, Zheng S, Li W, Huang B, Wang X. Regulation of plant 
water loss by manipulating the expression of phospholipase Dalpha. Plant J 2001; 28:135-144.

38 Zhao J, Wang X. Arabidopsis phospholipase D $\alpha 1$ interacts with the heterotrimeric G-protein $\alpha$-subunit through a motif analogous to the DRY motif in G-protein-coupled receptors. J Biol Chem 2004; 279:1794-1800.

39 Elias M, Potocky M, Cvrckova F, Zarsky V. Molecular diversity of phospholipase D in angiosperms. BMC Genomics 2002; 3:2.

40 Kusner DJ, Barton JA, Qin C, Wang X, Iyer SS. Evolutionary conservation of physical and functional interactions between phospholipase D and actin. Arch Biochem Biophys 2003; 412:231-241.

41 Li M, Qin C, Welti R, Wang X. Double knockouts of phospholipases Dzeta1 and Dzeta2 in Arabidopsis affect root elongation during phosphate-limited growth but do not affect root hair patterning. Plant Physiol 2006; 140:761-770.

42 Li M, Welti R, Wang X. Quantitative profiling of Arabidopsis polar glycerolipids in response to phosphorus starvation. Roles of phospholipases D zeta1 and D zeta2 in phosphatidylcholine hydrolysis and digalactosyldiacylglycerol accumulation in phosphorus-starved plants. Plant Physiol 2006; 142:750-761.

43 Alfandari D, Darribere TA. Simple PCR method for screening cDNA libraries. PCR Meth Appl 1994; 4:46-49.

44 Liu W, Xu ZH, Luo D, Xue HW. Roles of OsCKI1, a rice casein kinase I, in root development and plant hormone sensitivity. Plant J 2003; 36:189-202.

45 Kikuchi S, Satoh K, Nagata T, et al. Collection, mapping, and annotation of over 28,000 cDNA clones from japonica rice. Science 2003; 301:376-379.

46 Gomez-Cadenas A, Verhey SD, Holappa LD, Shen Q, Ho TH, Walker-Simmons MK. An abscisic acid-induced protein kinase, PKABA1, mediates abscisic acid-suppressed gene expression in barley aleurone layers. Proc Natl Acad Sci USA 1999; 96:17671772.

47 Gomez-Cadenas A, Zentella R, Walker-Simmons MK, Ho TH. Gibberellin/abscisic acid antagonism in barley aleurone cells: site of action of the protein kinase PKABA1 in relation to gibberellin signaling molecules. Plant Cell 2001; 13:667-679.

48 Shen Q, Gomez-Cadenas A, Zhang P, Walker-Simmons MK, Sheen J, Ho TH. Dissection of abscisic acid signal transduction pathways in barley aleurone layers. Plant Mol Biol 2001; 47:437448.

49 Yamauchi D, Zentella R, Ho TH. Molecular analysis of the barley (Hordeum vulgare L.) gene encoding the protein kinase PKABA1 capable of suppressing gibberellin action in aleurone layers. Planta 2002; 215:319-326.

50 Kobayashi Y, Yamamoto S, Minami H, Kagava Y, Hattori T. Differential activation of the rice sucrose nonfermenting 1 related protein kinase2 family by hyperosmotic stress and abscisic acid. Plant Cell 2004; 16:1163-1177.

51 Yoshida R, Hobo T, Ichimura K, et al. ABA-activated SnRK2 protein kinase is required for dehydration stress signaling in Arabidopsis. Plant Cell Physiol 2002; 43:1473-1483.

52 McGee JD, Roe JL, Sweat TA, Wang X, Guikema JA, Leach JE. Rice phospholipase D isoforms show differential cellular location and gene induction. Plant Cell Physiol 2003; 44:1013-1026.

53 Yamaguchi T, Tanabe S, Minami E, Shibuya N. Activation of phospholipase D induced by hydrogen peroxide in suspensioncultured rice cells. Plant Cell Physiol 2004; 45:1261-1270.

54 Yamaguchi T, Minami E, Ueki J, Shibuya N. Elicitor-induced activation of phospholipases plays an important role for the induction of defense responses in suspension-cultured rice cells. Plant Cell Physiol 2005; 46:579-587.

55 Bewley JD. Seed germination and dormancy. Plant Cell 1997; 9:1055-1066.

56 Finch-Savage WE, Leubner-Metzger G. Seed dormancy and the control of germination. New Phytol 2006; 171:501-523.

57 Yoshida R, Umezawa T, Mizoguchi T, Takahashi S, Takahashi F, Shinozaki K. The regulatory domain of SRK2E/OST1/SnRK2.6 interacts with ABI1 and integrates abscisic acid (ABA) and osmotic stress signals controlling stomatal closure in Arabidopsis. J Biol Chem 2006; 281:5310-5318.

58 Testerink C, Dekker HL, Lim ZY, et al. Isolation and identification of phosphatidic acid targets from plants. Plant J 2004; 39:527-536.

(Supplementary information is linked to the online version of the paper on the Cell Research website.) 